\title{
Isoscalar neutron-proton pairing and SU(4)-symmetry breaking in Gamow-Teller transitions
}

\author{
K. Kaneko ${ }^{1}$, Y. Sun ${ }^{2,3,4}$, T. Mizusaki ${ }^{5}$ \\ ${ }^{1}$ Department of Physics, Kyushu Sangyo University, Fukuoka 813-8503, Japan \\ ${ }^{2}$ School of Physics and Astronomy, Shanghai Jiao Tong University, Shanghai 200240, China \\ ${ }^{3}$ Collaborative Innovation Center of IFSA, Shanghai Jiao Tong University, Shanghai 200240, China \\ ${ }^{4}$ Institute of Modern Physics, Chinese Academy of Sciences, Lanzhou 730000, China \\ ${ }^{5}$ Institute of Natural Sciences, Senshu University, Tokyo 101-8425, Japan
}

(Dated: May 17, 2018)

\begin{abstract}
The isoscalar neutron-proton pairing is thought to be important for nuclei with equal number of protons and neutrons but its manifestation in structure properties remains to be understood. We investigate the Gamow-Teller (GT) transitions for the $f_{7 / 2}$-shell nuclei in large-scale shell-model calculations with the realistic Hamiltonian. We show that the isoscalar $T=0, J^{\pi}=1^{+}$neutron-proton pairing interaction plays a decisive role for the concentration of GT strengths at the first-excited $1_{1}^{+}$state in ${ }^{42} \mathrm{Sc}$, and that the suppression of these strengths in ${ }^{46} \mathrm{~V},{ }^{50} \mathrm{Mn}$, and ${ }^{54} \mathrm{Co}$ is mainly caused by the spin-orbit force supplemented by the quadrupole-quadrupole interaction. Based on the good reproduction of the charge-exchange reaction data, we further analyze the interplay between the isoscalar and isovector pairing correlations. We conclude that even for the most promising $A=42$ nuclei where the SU(4) isoscalar-isovector-pairing symmetry is less broken, the probability of forming an isoscalar neutron-proton pairing condensation is less than $60 \%$ as compared to the expectation at the SU(4)-symmetry limit.
\end{abstract}

PACS numbers: 21.30.Fe, 21.60.Cs, 21.10.Dr, 27.50.+e

Introduction. Pairing of two kinds of fermions is a unique phenomenon in nuclear physics. According to Heisenberg [1], neutrons and protons can be regarded as two "states" of nucleons, described by isospin $T$. Thus, nuclear many-body states and interactions are characterized by combinations of spin and isospin [2], respecting the antisymmetrization requirement for wave functions. For most nuclei with $N>Z$, the isovector $(T=1)$ neutron-neutron $(n n)$ and proton-proton $(p p)$ pairings coupled to angular momentum zero $(J=0)$ can be well described by the BCS-type models [3-5] similarly as in condensed-matter physics. For nuclei near the $N=Z$ line, the neutrons and protons close to the Fermi surface occupy identical orbits, and thus can have large probabilities to form isovector neutron-proton $(n p)$ pairs with $T=1$ or isoscalar $(T=0) n p$ pairs. With a short-range force, the angular momentum of these $n p$ pairs favors $J=0$ for $T=1$, and $J=1$ or $J=J_{\max }$ for $T=0$ [6]. It has been shown that the $n p$ pairing with $T=1, J=0$ should be treated on an equal footing with $T=1, J=0 n n$ and $p p$ pairing [7].

The isoscalar $T=0 \mathrm{np}$ pairing, on the other hand, is special in nuclear physics, and has long been a discussion focus. An interesting question has been whether the $T=0 \mathrm{np}$ pairing condensate can occur in nuclei. $\beta$-decay and chargeexchange reaction, which involve isospin-flip, are thought to be the means to study the $T=0 n p$-pairing [6]. In the recent $\left({ }^{3} \mathrm{He}, t\right)$ charge-exchange experiments, Fujita et al. [8, 9] have shown an interesting observation in the ${ }^{42} \mathrm{Ca} \rightarrow{ }^{42} \mathrm{Sc}$ reaction. They found that, instead of the usual expectation for nuclei with mass $A>60$ that most of Gamow-Teller (GT) strengths are distributed with a few $\mathrm{MeV}$ width at $E_{x}>9 \mathrm{MeV}$ [10], the GT strengths they obtained concentrate in the lowest excited $1^{+}$state at $0.6 \mathrm{MeV}$ in ${ }^{42} \mathrm{Sc}$, which they call the lowenergy super GT state. Moreover, in the same reaction for all other $f_{7 / 2}$-shell nuclei, which ends up with the odd-odd $N=Z$
$\left(T_{z}=0\right)$ nuclei (i.e., ${ }^{46} \mathrm{~V},{ }^{50} \mathrm{Mn}$, and ${ }^{54} \mathrm{Co}$ ), the GT strength distributions are found to be qualitatively different. As mass number $A$ increases, the low-energy strength becomes fragmented and the bumplike structure in the high-energy region begins to develop. Finally in ${ }^{54} \mathrm{Co}$, the distribution is mainly of the typical bumplike GT resonances. These results seem to suggest drastic differences occurring along the $N=Z$ line, which have attracted our attention.

Combining the $T=0, J^{\pi}=1^{+}$mode with the $T=1, J^{\pi}=$ $0^{+}$one, the isovector and isoscalar pairing correlations have been examined by the exactly solvable $\mathrm{SO}(8)$ model with degenerate single-particle orbits [11, 12]. In the SU(4) symmetry limit, the GT strength is, as pointed out by Wigner [13], indeed expected to concentrate at the lowest $1^{+}$state. Thus the large GT strength at the lowest $1^{+}$state observed in ${ }^{42} \mathrm{Sc}$ [8,9] would correspond to an SU(4) symmetry, and it could be the fingerprint of the $T=0$ pairing. The GT strength distributions with $A=42-58$ were studied by using a self-consistent Skyrme Hartree-Fock-Bogoliubov plus quasiparticle randomphase approximation (QRPA) method including isoscalar and isovector residual interactions [14]. In Ref. [15], Yoshida considered the lowest $1_{1}^{+}$in ${ }^{42} \mathrm{Sc}$ as a precursory soft mode of the $T=0$ pairing condensation. There was reported experiment for the GT decay to the odd-odd $N=Z^{62}$ Ga suggesting no evidence of the isoscalar pairing condensation [16]. It is important to mention that the SU(4) symmetry can be largely suppressed due to the presence of the spin-orbit (SO) splitting [17, 18]. The isoscalar spin-triplet pairing interaction is significant for the enhancement of the GT strength, while the experimental evidence of the $T=0$ pairing condensation is still controversial [6, 19].

Thus, despite the great efforts made in the past, both experimentally and theoretically, there has been no consensus on the $T=0 n p$-pairing condensation in nuclei. A realistic model 
that describes the shell effect and contains all kinds of relevant interactions, which either favor or unfavor the $n p$-pairing condensate, could shed light on this question.

The PMMU model. The present authors have recently proposed a unified realistic shell-model Hamiltonian called PMMU [20, 21], consisting of the two-body interactions in separable forms with the monopole interaction $V_{m}^{M U}$ constructed from the monopole-based universal force [22],

$$
\begin{aligned}
H= & H_{0}+H_{P M}+H_{m}^{M U} \\
H_{0}= & \sum_{\alpha} \varepsilon_{a} c_{\alpha}^{\dagger} c_{\alpha} \\
H_{P M}= & -\frac{1}{2} \sum_{J=0,2} g_{J} \sum_{M \kappa} P_{J M 1 \kappa}^{\dagger} P_{J M 1 \kappa}-\frac{1}{2} \chi_{2} \sum_{M}: Q_{2 M}^{\dagger} Q_{2 M}: \\
& -\frac{1}{2} g_{1} \sum_{M} P_{1 M 00}^{\dagger} P_{1 M 00} \\
H_{m}^{M U}= & \sum_{a \leq b, T} V_{m}^{M U}(a b, T) \sum_{J M K} A_{J M T K}^{\dagger}(a b) A_{J M T K}(a b)
\end{aligned}
$$

It was demonstrated [20] that the PMMU interaction can well describe nuclear properties of the $p f$ and $p f_{5 / 2} g_{9 / 2}$ shell nuclei. In Eq. (3), the first term denotes the isovector $J=0$ pairing $(P 01)$ and $J=2$ pairing $(P 21)$ interactions in the particle-particle channel, and the second term the quadrupole-quadrupole $(Q Q)$ interaction in the particlehole channel. The isoscalar $J=1$ pairing $(P 10)$ interaction in the particle-particle $T=0$ channel is added into the PMMU Hamiltonian as the third term in Eq. (3). The monopole interaction $H_{m}^{M U}$ is constructed from the monopole-based universal force [22], and its effect has been extensively discussed in Refs. [20, 21].

In the present work, we use the same single-particle energies $\varepsilon_{a}$ and the parameter strengths of our previous paper [20] for the $p f$ shell space. The spin-orbit splitting $6.5 \mathrm{MeV}$ between the spin-orbit parter $f_{7 / 2}$ and $f_{5 / 2}$ is chosen so as to fit the measured value in ${ }^{41} \mathrm{Ca}$. The isoscalar monopole terms are slightly shifted so as to fit the observed lowest $1_{1}^{+}$energies for ${ }^{46} \mathrm{~V},{ }^{50} \mathrm{Mn}$, and ${ }^{54} \mathrm{Co}$. For the added $T=0, J=1$ pairing interaction, the force strength is given by $g_{1}=g_{1}^{0} / A$, which has the same mass-dependence as the $T=1, J=0$ interaction. It should be mentioned that there is no common agreement for the choice of $g_{1}$ [15, 23]. We have confirmed that this additional $P 10$ force does not change the results and conclusions obtained in our previous paper [20]. Shell-model calculations are performed with the code MSHELL64 [24].

On the $S U(4)$-symmetry breaking. Figure 1 shows the calculated $\mathrm{B}(G T)$ distributions and the experimental results in the ${ }^{42} \mathrm{Ca} \rightarrow{ }^{42} \mathrm{Sc}$ reaction. The quenching factor $q=0.74$ is used for the GT-strength calculation as it proves to be appropriate for the mass region of $A \sim 40-60$ [25]. We can see that the $P 10$ pairing interaction with the parameter $g_{1}^{0}=12.5 \mathrm{MeV}$ reproduces the $\mathrm{B}(G T)$ data as well as the lowest $1_{1}^{+}$excitation energy. With weaker $g_{1}^{0}$, the GT strength is suppressed, and the lowest $1^{+}$excitation is shifted to higher energies. This demonstrates clearly that a sufficiently strong isoscalar $P 10$

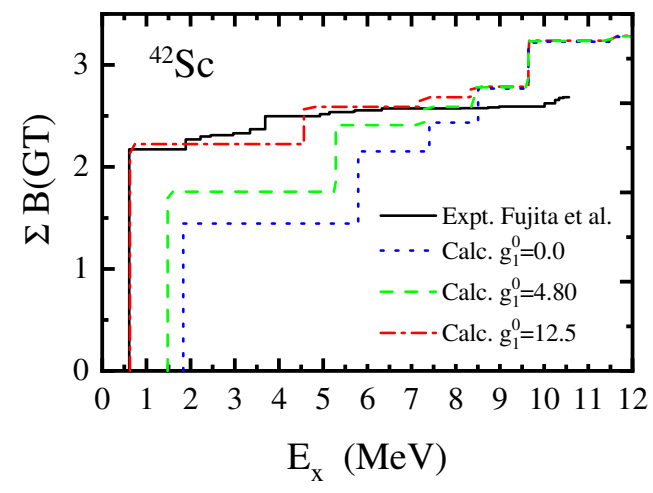

FIG. 1: (Color online) Cumulative sums of $\mathrm{B}(\mathrm{GT})$ in ${ }^{42} \mathrm{Sc}$, calculated by the PMMU Hamiltonian including the $T=0, J=1$ interaction with strengths $g_{1}^{0}=0.0,4.80$, and 12.5 , and compared with the experimental data from Ref. [9].

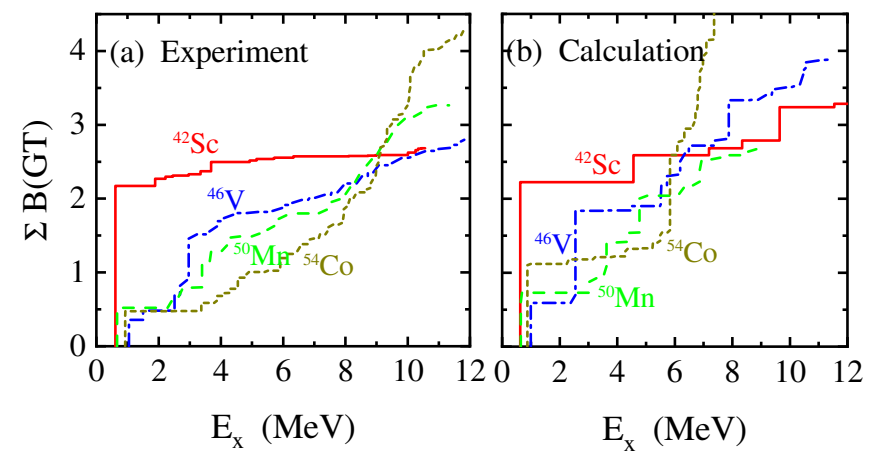

FIG. 2: (Color online) Cumulative sums of $\mathrm{B}(G T)$ of the daughter nuclei ${ }^{42} \mathrm{Sc},{ }^{46} \mathrm{~V},{ }^{50} \mathrm{Mn}$, and ${ }^{54} \mathrm{Co}$ for (a) experimental data [9] and (b) calculation.

pairing interaction is essential for the occurrence of the lowenergy super GT state observed in Refs. [8, 9]. The obtained sudden increase of the GT strength at $E_{x}=9.64 \mathrm{MeV}$ for all calculations with different $P 10$ pairing strengths is due to the $T=1$ states. Previously the shell-model calculation using the GXPF1J interaction [26] was performed to study the same data [8, 9]. The predicted $1_{1}^{+}$excitation energy $0.33 \mathrm{MeV}$ was about half of the experimental energy $0.611 \mathrm{MeV}$, while the GT distribution was reproduced well.

Figure 2(a) summarizes the experimental $\mathrm{B}(G T)$ distributions [9] as functions of excitation energies of the daughter nuclei ${ }^{42} \mathrm{Sc},{ }^{46} \mathrm{~V},{ }^{50} \mathrm{Mn}$, and ${ }^{54} \mathrm{Co}$, in which strong massdependence is evident. The GT strength concentrates in the lowest $1_{1}^{+}$state of ${ }^{42} \mathrm{Sc}$, but moves to higher energies with more spread patterns for ${ }^{46} \mathrm{~V},{ }^{50} \mathrm{Mn}$, and ${ }^{54} \mathrm{Co}$. The calculated results in Fig. 2 (b) show qualitatively the same trend.

Our employed shell-model Hamiltonian in Eq. (1) is rich in physics, which contains all relevant interactions in the form of separable forces. The $H_{0}$ term and the monopole interaction $H_{m}^{M U}$ produce the realistic single-particle states, ensuring that the SO effect is correctly described. In the particle-particle channel, the $P 10, P 21$, and $P 01$ terms present the competi- 
tion of isoscalar and isovector pairing, and in the particle-hole channel, $Q Q$ describes the correlation induced by deformation. If we assume the degenerate single-particle orbits and take only the $T=1, J=0$ and $T=0, J=1$ pairing interactions in the PMMU, it reduces to the solvable $\mathrm{SO}(8)$ model [11, 12]. Further, if the $T=0$ and $T=1$ pairing force strengths are set to be equal, the Hamiltonian is invariant under SU(4), in which the GT strength to the lowest $1^{+}$state becomes large [13]. In turn, the appearance of the large GT strength at the lowest $1^{+}$state implies that both pairing correlations are equally significant. However, it has been discussed that this large GT value is suppressed due to strong SO splitting [18]. Thus it is very interesting to study the contribution of various terms in the realistic PMMU Hamiltonian to the GT strengths, to answer the question whether and under which conditions the isoscalar $n p$ pairing can lead to a collective mode coexisting with that of the isovector pairing.

In Fig. 3, the effect of each term in Eq. (1) is studied with the $\mathrm{B}(G T)$ for the first excited $1_{1}^{+}$states in the $A=42-54$ daughter nuclei. If $Q Q$ and $P 21$ in Eq. (3) and the monopole $H_{m}^{M U}$ interaction in Eq. (4) are all switched off and the SO splitting is excluded from the calculation, Eq. (1) becomes the Hamiltonian with only the isoscalar $P 10$ and isovector $P 01$ pairing terms. Further, if the strengths of both pairing terms are set to be equal, i.e., $g_{1}=g_{0}$, then it is invariant under SU(4). In the limit of the exact SU(4) symmetry, the GT strength is concentrated in a large single transition to the lowest $1_{1}^{+}$GT state [13]. Since the second term in the Ikeda sum rule for the GT strength, $\sum B\left(G T_{-}\right)-\sum B\left(G T_{+}\right)=3(N-Z) q^{2}$, can be neglected, the SU(4) symmetry corresponds to a constant GT strength $B(G T)=6 q^{2} \approx 3.29$ for all cases of $A=$ $42-54$, as shown in Fig. 3

The departure from the SU(4) symmetry limit occurs as these terms are put back. The SO splitting in the $p f$-shell is known to be sizable, giving rise to the magic number 28 . The realistic SO force leads to the SU(4) symmetry breaking and suppression of the GT strengths. As seen in Fig. [3. the largest $\mathrm{SO}$ effect is found at $A=54$, where about $2 / 3$ of the SU(4) value is suppressed. Among the four cases, $A=42$ has the smallest influence. On the other hand, the inclusion of the $J=2$ pairing does not bring much effect to GT. However, when the $Q Q$ interaction is switched on, the $\mathrm{B}(G T)$ for $A=46,50$ are drastically reduced, while the effect on $A=42,54$ is smaller. Thus the $Q Q$ interaction is largely responsible for the small $\mathrm{B}(G T)$ values for $A=46$ and 50 . With the monopole interactions included and $g_{1}^{0}=0.26$, finally the realistic PMMU Hamiltonian reproduces very well the experimental data. Only for $A=54$, the calculated result is somewhat larger than the data.

Question on the isoscalar neutron-proton pairing condensation. One important finding in Fig. 3 is that the $\mathrm{B}(G T)$ of $A=42$ has the weakest breaking of the SU(4) symmetry, and it is caused mainly by the SO splitting. In an even-even nucleus with the isovector $J=0$ pairing interaction, the ground state with seniority zero is usually interpreted as a condensate of isovector $J=0$ pairs. Then the isoscalar pairing conden-

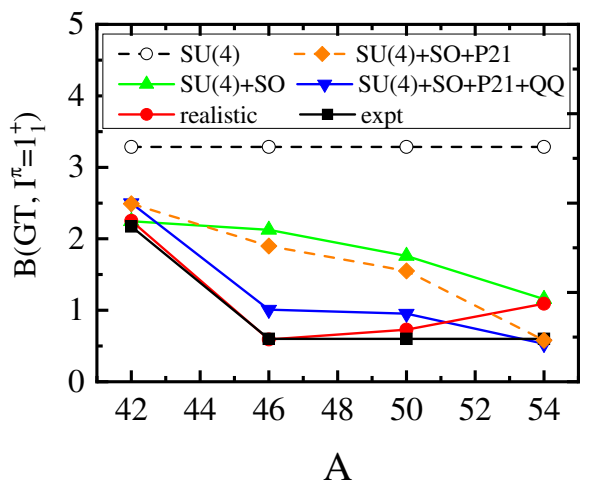

FIG. 3: (Color online) Calculated $\mathrm{B}(G T)$ strengths of the lowest $1_{1}^{+}$ states in the daughter nuclei ${ }^{42} \mathrm{Sc},{ }^{46} \mathrm{~V},{ }^{50} \mathrm{Mn}$, and ${ }^{54} \mathrm{Co}$ with separate contributions from each interaction in Eqs. (2)-(4), compared with the experimental data [8] and the value of the SU(4) symmetry.

sate may be realized for the isoscalar $J=1$ pairing Hamiltonian with the degenerate single-particle orbits. However, this isoscalar pairing condensate may not persist against the single-particle splitting due to the SO force [18]. With all these factors taking into account, we formulate an isoscalarisovector-pairing Hamiltonian

$H=H_{0}-\frac{g(1-x)}{2} \sum_{\kappa} P_{001 \kappa}^{\dagger} P_{001 \kappa}-\frac{g(1+x)}{2} \sum_{M} P_{1 M 00}^{\dagger} P_{1 M 00}$,

with $x$ being a control parameter. Equation (5) can be regarded as a simplified model from the realistic PMMU Hamiltonian (1) to study the competition of isoscalar and isovector pairings under the presence of the SO splitting. If the single-particle energies in $H_{0}$ are degenerate, the Hamiltonian (5) is invariant under $\mathrm{SO}(8)$, and $x=-1$ and $x=1$ corresponds, respectively, to the usual isovector $J=0$ pairing and isoscalar $J=1$ pairing Hamiltonian. For $x=0$, the Hamiltonian (5) is invariant under SU(4). Thus by varying $x$, it describes the phase evolution from the $T=1, J=0$ pairing phase $(x=-1)$, through the $\mathrm{SU}(4)$ Wigner super-multiplet phase ( $x=0)$, to the $T=0, J=$ 1 pairing phase $(x=1)$.

Figure 4 shows the calculated results obtained by solving the Hamiltonian (5), where the non-degenerate realistic single-particle energies [20] are employed. The competition between the lowest $0_{1}^{+}$and $1_{1}^{+}$states to be the ground state are shown in Fig. $4(\mathrm{a})$ for the $T_{z}=0$ nuclei ${ }^{42} \mathrm{Sc},{ }^{46} \mathrm{~V},{ }^{50} \mathrm{Mn}$, and ${ }^{54} \mathrm{Co}$. It is seen that for all these odd-odd $N=Z$ nuclei, $0_{1}^{+}$is definitely the lowest state for all negative $x$ 's and small positive $x$ 's. Starting from $x=-1$, all the $1_{1}^{+}$energies drop continuously with increasing $x$, and are inverted in position with the $0_{1}^{+}$states at a critical point around $x_{c}=0.20$, after which the $0_{1}^{+}$energies increase. For ${ }^{42} \mathrm{Sc}, x_{c}=0.17$. A correlated result is shown in Fig. 4(b), where from $x=-1$, the calculated $\mathrm{B}(G T)$ transition from ${ }^{42} \mathrm{Ca}$ to the daughter ${ }^{42} \mathrm{Sc}$ increases with $x$, and reaches the maximum at $x_{c}=0.17$.

There has been a long-standing question whether a strong 


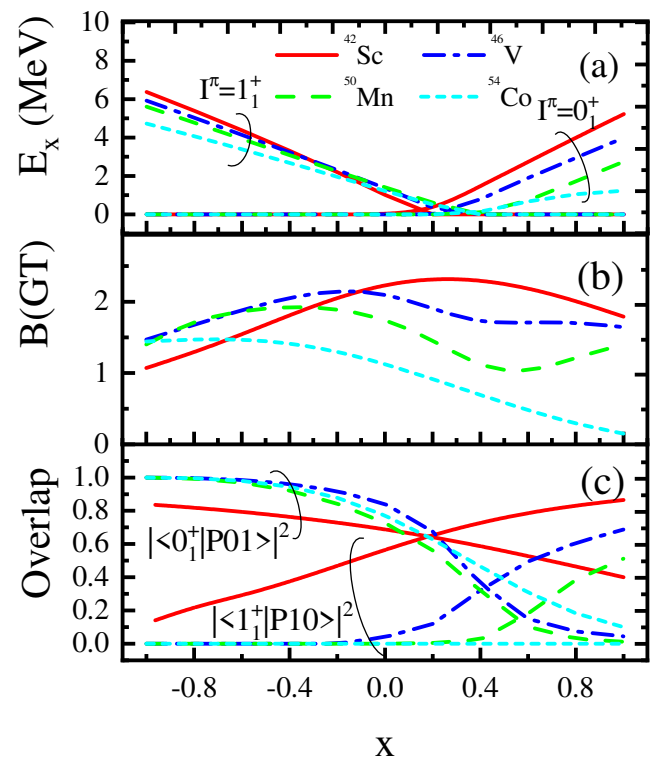

FIG. 4: (Color online) Excitation energy, B $(G T)$ value, and overlap between the wavefunctions by solving Eq. (5) and the realistic Hamiltonian (1), as functions of the control parameter $x$.

isoscalar pairing correlation can lead to condensation. The possibility of finding the isoscalar pairing condensate was theoretically investigated for heavy nuclei [18]. We note that the Hamiltonian in the present work is superior to that used in Ref. [18]. To see what our shell model calculation may suggest, we follow the definition for the isovector and isoscalar pairing condensates in Refs. [17, 27]. The isovector $J=0$ pairing condensation state $|P 01\rangle$ is obtained by solving the second term and the first term with experimental single-particle energies in Eq. (5). The isoscalar $J=1$ pairing condensate state $|P 10\rangle$ is obtained by solving the third term and the first term with degenerate single-particle energies. The probabilities of finding a nuclear state in different condensed phases are given by the squared overlap of that state with $|P 01\rangle$ or $|P 10\rangle$.

In Fig. 4( $)$, the squared overlaps $\left|\left\langle 0_{1}^{+} \mid P 01\right\rangle\right|^{2}$ and $\left|\left\langle 1_{1}^{+} \mid P 10\right\rangle\right|^{2}$ are shown as functions of $x$ in Eq. (5). For all the four $f_{7 / 2}$-shell odd-odd $N=Z$ nuclei, $\left|\left\langle 0_{1}^{+} \mid P 01\right\rangle\right|^{2}$ is overwhelmingly large when $x$ is negative. Beyond $x=0$, it begins to decrease with positive $x$ 's. At $x=+1$, this quantity is close to zero except for ${ }^{42} \mathrm{Sc}$. On the other hand, the probability of finding the isoscalar pair condensate in the $1_{1}^{+}$ state of ${ }^{46} \mathrm{~V},{ }^{50} \mathrm{Mn}$, and ${ }^{54} \mathrm{Co}$ is zero for the negative $x$ 's. As $x$ varies from -1 to +1 , the overlap $\left|\left\langle 1_{1}^{+} \mid P 10\right\rangle\right|^{2}$ for ${ }^{42} \mathrm{Sc}$ increases monotonously with no drastic changes, and $\left|1_{1}^{+}\right\rangle$ evolves smoothly from the isovector into the isoscalar pairing phase. At $x=0$ (the SU(4) symmetry limit), the overlaps of the condensation states with the physical ground $0_{1}^{+}$and excited $1_{1}^{+}$states of ${ }^{42} \mathrm{Sc}$ are obtained as $\left|\left\langle 0_{1}^{+} \mid P 01\right\rangle\right|^{2}=0.99$ and $\left|\left\langle 1_{1}^{+} \mid P 10\right\rangle\right|^{2}=0.57$, respectively.

In sharp contrast, the overlap $\left|\left\langle 1_{1}^{+} \mid P 10\right\rangle\right|^{2}$ for ${ }^{46} \mathrm{~V}$ and ${ }^{50} \mathrm{Mn}$ begins to take a nonzero value only when $x$ is positive, and for ${ }^{54} \mathrm{Co},\left|\left\langle 1_{1}^{+} \mid P 10\right\rangle\right|^{2}$ is always zero for any positive $x$. In partic- ular, with the realistic parameter around $x=0$ corresponding to the SU(4) symmetry, $\left|\left\langle 1_{1}^{+} \mid P 10\right\rangle\right|^{2}$ is zero for ${ }^{46} \mathrm{~V},{ }^{50} \mathrm{Mn}$, and ${ }^{54} \mathrm{Co}$. This means that for the four cases in the $f_{7 / 2^{-}}$ shell, it is impossible to realize an isoscalar pairing condensation. In ${ }^{42} \mathrm{Sc}$, the calculated probability $\left|\left\langle 1_{1}^{+} \mid P 10\right\rangle\right|^{2}$ is $57 \%$. The formed isoscalar $J=1$ pairing coexists with the isovector $J=0$ pairing, while ${ }^{42} \mathrm{Sc}$ contains only one valence $n p$ pair.

Summary. In conclusion, to investigate the role of the isoscalar $n p$ pairing interaction in GT transitions and the possibility of $n p$ pairing condensation in nuclei, we performed large-scale shell-model calculations with the realistic PMMU Hamiltonian for the $f_{7 / 2}$-shell nuclei. The early works based on simpler models [28, 29] are close in spirit to the present work. The isoscalar $T=0, J=1$ interaction is found to be decisively important for explaining the large $\mathrm{B}(G T)$ strengths of the transition from the ground $0_{1}^{+}$state in ${ }^{42} \mathrm{Ca}$ to the lowest $1_{1}^{+}$state in ${ }^{42} \mathrm{Sc}$. The systematics of $\mathrm{B}(G T)$ distributions are well described for the mass $A=42,48,50,54$. Our realistic shell-model calculation with more realistic interactions confirms the previous conclusion [18] that the $B(G T)$ strength in these nuclei is considerably suppressed by the SO splitting and the SU(4) symmetry is broken. We also show that the $B(G T)$ strengths are largely suppressed by the $Q Q$ interaction for the masses $A=46$ and 50. The deformation effect in GT transitions was emphasized in Ref. [30]. We discussed the isovector and isoscalar pairing condensation using a simplified model reduced from the PMMU Hamiltonian. The results suggest that even for the most promising $A=42$ nuclei where the $\mathrm{SU}(4)$ isoscalar-isovector-pairing symmetry is less broken, the probability of forming an isoscalar $n p$ pairing condensate is less than $60 \%$ as compared to the idealized situation. Finally, we note that the present realistic shell-model calculation may be applied to the study of SU(4)-symmetry in double- $\beta$ decay, as discussed recently in Ref. [31].

Acknowledgement. One of us (YS) thanks Y. Fujita for valuable discussions during his visit in Shanghai. This work was partially supported by the National Natural Science Foundation of China (No. 11575112) and by the National Key Program for S\&T Research and Development (No. 2016YFA0400501).

[1] W. Heisenberg, Z. Phys. 77, 1 (1932).

[2] F. Osterfeld, Rev. Mod. Phys. 64, 491 (1992).

[3] J. Bardeen, L. Cooper and J. R. Schrieffer, Phys. Rev. 108, 1175 (1957).

[4] A. Bohr, B. R. Mottelson, and D. Pines, Phys. Rev. 110, 936 (1958).

[5] S. T. Belyaev, Mat. Fys. Medd. Dan. Vid. Selsk. 31 (no. 11), (1959).

[6] S. Frauendorf and A. O. Machiavelli, Prog. Part. Nucl. Phys. 78, 24 (2014).

[7] S. Frauendorf and J. A. Sheikh, Nucl. Phys. A 645, 509 (1999); Phys. Rev. C 59, 1400 (1999).

[8] Y. Fujita et al., Phys. Rev. Lett. 112, 112502 (2014).

[9] Y. Fujita et al., Phys. Rev. C 91, 064316 (2015). 
[10] J. Rapaport and E. Sugarbaker, Annu. Rev. Nucl. Part. Sci. 44, 109 (1994).

[11] J. Engel, S. Pittel, M. Stoitsov, P. Vogel, and J. Dukelsky, Phys. Rev. C 55, 1781 (1997).

[12] J. A. Evans, G. G. Dussel, E. E. Maqueda, and R. P. J. Perazzo, Nucl. Phys. A 367, 77 (1981).

[13] E. Wigner, Phys. Rev. 51, 106 (1937).

[14] C. L. Bai, H. Sagawa, G. Colo, Y. Fujita, H. Q. Zhang, X. Z. Zhang, and F. R. Xu, Phys. Rev. C 90, 054335 (2014).

[15] K. Yoshida, Phys. Rev. C 90, 031303(R) (2014).

[16] E. Grodner et al., Phys. Rev. Lett. 106, 252502 (2011).

[17] A. Poves and G. Martinez-Pinedo, Phys. Lett. B 430, 203 (1998).

[18] G. F. Bertsch and Y. Luo, Phys. Rev. C 81, 064320 (2010).

[19] A. O. Machiavelli et al., Phys. Lett. B 480, 1 (2000).

[20] K. Kaneko, T. Mizusaki, Y. Sun, and S. Tazaki, Phys. Rev. C 89, 011302(R) (2014).

[21] K. Kaneko, T. Mizusaki, Y. Sun, and S. Tazaki, Phys. Rev. C 92, 044331 (2015).
[22] T. Otsuka, T. Suzuki, M. Honma, Y. Utsuno, N. Tsunoda, K. Tsukiyama, and M. Hjorth-Jensen, Phys. Rev. Lett. 104, 012501 (2010).

[23] N. Hinohara and J. Engel, Phys. Rev. C 90, 031301(R) (2014).

[24] T. Mizusaki, N. Shimizu, Y. Utsuno, and M. Honma, code MSHELL64 (unpublished).

[25] G. Martinez-Pinedo, A. Poves, E. Caurier, and A. P. Zuker, Phys. Rev. C 53, R2602 (1996).

[26] M. Honma, T. Otsuka, T. Mizusaki, M. Hjorth-Jensen, and B. A. Brown, J. Phys. Conf. Ser. 20, 7 (2005).

[27] M. Dufour and A. P. Zuker, Phys. Rev. C 54, 1641 (1996).

[28] D. Cha, Phys. Rev. C 27, 2269 (1983).

[29] E. Moya de Guerra, A. A. Radutab, L. Zamick, and P. Sarriguren, Nucl. Phys. A 727, 3 (2003).

[30] I. Stetcu and C. W. Johnson, Phys. Rev. C 69, 024311 (2004).

[31] V. dos S. Ferreira, F. Krmpotić, C. A. Barbero, and A. R. Samana, Phys. Rev. C 96, 044322 (2017). 